\title{
Comparison of surgical fixation and non-operative management in patients with traumatic sternum fracture
}

\author{
Ashton B. Christian ${ }^{1}$ (1) $\cdot$ Areg Grigorian ${ }^{2} \cdot$ Jeffry Nahmias $^{1} \cdot$ William Q. Duong $^{1} \cdot$ Michael Lekawa $^{1} \cdot$ Victor Joe $^{1}$. \\ Matthew Dolich ${ }^{1} \cdot$ Sebastian D. Schubl ${ }^{1}$
}

Received: 27 May 2020 / Accepted: 9 October 2020 / Published online: 19 October 2020

○) Springer-Verlag GmbH Germany, part of Springer Nature 2020

\begin{abstract}
Purpose The incidence of sternal fractures in blunt trauma patients lies between 3 and $7 \%$. The role, timing and indications for surgical management are not well delineated and remain controversial for patients undergoing surgical stabilization of sternum fracture (SSSF). We sought to identify the national rate of SSSF in patients with a sternum fracture hypothesizing patients undergoing SSSF will have a decreased rate of mortality and complications.

Methods The Trauma Quality Improvement Program (2015-2016) was queried for patients with sternum fracture. Propensity scores were calculated to match patients undergoing SSSF to patients managed non-operatively in a 1:2 ratio using demographic data.

Results From 9460 patients with a sternum fracture, 114 (1.2\%) underwent SSSF. After propensity-matching, 112 SSSF patients were compared to 224 patients undergoing non-operative management (NOM). There were no differences in matched characteristics (all $p>0.05$ ). Compared to patients undergoing NOM, patients undergoing SSSF had an increased median length of stay (LOS) (16 vs. 7 days, $p<0.001$ ), ICU LOS (9.5 vs. 5.5 days, $p=0.016)$ and ventilator days ( 8 vs. $5, p=0.035$ ). The SSSF group had a similar rate of ARDS (2.7\% vs. $2.2 \%, p=0.80)$, pneumonia $(1.8 \%$ vs. $0.9 \%, p=0.48)$ and unplanned intubation $(8.9 \%$ vs. $5.8 \%, p=0.29)$ but a lower mortality rate $(2.7 \%$ vs. $11.2 \%, p=0.008)$.

Conclusion Just over $1 \%$ of patients with sternum fracture underwent SSSF in a national analysis. Patients undergoing SSSF had an increased LOS and similar rate of all measured pulmonary complications, however a lower mortality rate compared to patients managed non-operatively.
\end{abstract}

Keywords Sternum $\cdot$ Sternal $\cdot$ Fracture $\cdot$ Trauma $\cdot$ Stabilization $\cdot$ Fixation

Ashton B. Christian

ashtonbc@hs.uci.edu

Areg Grigorian

areg.grigorian@med.usc.edu

Jeffry Nahmias

jnahmias@hs.uci.edu

William Q. Duong

duongw@uci.edu

Michael Lekawa

melekawa@hs.uci.edu

Victor Joe

vcjoe@hs.uci.edu
Matthew Dolich

mdolich@hs.uci.edu

Sebastian D. Schubl

sschubl@hs.uci.edu

1 Department of General Surgery, Division of Trauma, Burns and Surgical Critical Care, University of California, Irvine, 333 City Blvd. West, Suite 1600, Orange, CA 92868, USA

2 Department of Surgery, Division of Trauma and Surgical Critical Care, University of Southern California, Los Angeles, CA, USA 


\section{Introduction}

The incidence of sternal fractures in blunt trauma patients is between 3 and 7\% with a small subset of patients failing conservative non-operative management (NOM) due to significant pain, respiratory compromise, and/or nonunion [1-6]. The morbidity and mortality associated with sternal fractures are mostly affected by concomitant thoracic injuries with mortality rates ranging from $4-45 \%$ $[2,4,5]$. Though the vast majority of sternal fractures can be successfully treated conservatively, approximately $6 \%$ (1-11\% range) will undergo surgical intervention often for non-union or a severe fracture with uncontrollable pain [1, 4-9]. However, no consensus guidelines exist regarding patient selection for surgical stabilization of sternum fracture (SSSF).

Surgical technology for such injuries has advanced significantly in recent decades since initial attempts at fixation were introduced in the 1940s [10]. Dedicated surgical systems now exist and there are improvements in conservative measures, such as analgesia, and passive reduction of displacement $[1,3,5,10]$. Despite such improvements, we continue to lack high-quality studies regarding SSSF. In other orthopedic injuries, such as clavicular fractures, technologic advancements have led to a shift toward increased use of operative management $[11,12]$.

Therefore, we sought to identify the national incidence of SSSF in patients with sternum fracture hypothesizing that patients undergoing SSSF would have a decreased rate of acute respiratory distress syndrome, pneumonia, unplanned intubation, and mortality.

\section{Methods}

This study was approved by the University of California, Irvine's institutional review board. We performed a retrospective analysis of the Trauma Quality Improvement Program (TQIP) between January 2015 and December 2016. Under the American College of Surgeons, TQIP includes over 850 trauma centers across the United States with the goal of elevating the quality of care provided to trauma patients. It accomplishes this by collecting data from trauma centers, providing feedback about performance, and identifying characteristics that individual trauma centers can improve upon. We identified all patients 18 years of age and older presenting with traumatic sternal fracture. Two groups were compared: trauma patients undergoing SSSF and patients undergoing NOM for traumatic sternum fractures. Propensity scores were calculated to match patients undergoing SSSF to patients managed $\mathrm{w}$
NOM in a 1:2 ratio using age, sex, comorbidities, trauma Injury Severity Score (ISS) and severe grade (>3) for the Abbreviated Injury Scale (AIS) of the head, thorax and abdomen. The specific comorbidities used for propensity matching included hypertension, chronic obstructive pulmonary disease, chronic renal failure, smoking status, and diabetes. The specific injuries used for propensity matching include pelvic fracture, rib fractures, upper/lower extremity fracture, spine fracture, thoracic vessel injury, heart injury, traumatic brain injury, splenic injury, liver injury, and lung injuries including hemothorax, pneumothorax, and unspecified lung injuries.

The primary outcome was mortality. Secondary outcomes included total hospital length of stay (LOS), intensive care unit (ICU) LOS, ventilator days, and in-hospital complications. In-hospital complications included acute kidney injury, acute respiratory distress syndrome, cardiac arrest, deep site infection, deep vein thrombosis, myocardial infarction, organ space infection, pneumonia, pulmonary embolism, cerebrovascular accident, superficial infection, unplanned intubation, urinary tract infection, unplanned return to the operating room, unplanned ICU admission, severe sepsis, catheter-associated urinary tract infection, ventilator-acquired pneumonia, and other unclassified complications.

All variables were coded as either present or absent. Descriptive statistics were performed for all variables. A Student's $t$ test or Mann-Whitney $U$ test was used to compare continuous variables and a chi-square was used to compare categorical variables for bivariate analysis. Categorical data were presented as percentages, and continuous data were reported as medians with interquartile range. All $p$-values were two-sided, with a statistical significance level of $<0.05$. All missing data points were not imputed but treated as missing data. All analyses were performed with IBM SPSS Statistics for Windows (Version 24, IBM Corp., Armonk, NY).

\section{Results}

\section{Demographics}

Of the 9460 patients with a sternum fracture, $114(1.2 \%)$ underwent SSSF, and after propensity matching, 112 SSSF patients were compared to the 224 matched patients undergoing NOM. Between the SSSF and NOM cohorts, there was no difference in age (50 vs. 49 ), male gender (75\% vs. $79.9 \%$ ), ISS (19 vs. 19 ), or any comorbidities ( $p>0.05$ for all) (Table 1). With regard to injury profiles, there were no differences between the two cohorts when considering AIS, or associated injuries (all $p>0.05$ ) (Table 1). Table 1 includes factors used for propensity 
Table 1 Comparison of factors between patients managed with NOM vs SSSF-propensity-matched

\begin{tabular}{|c|c|c|c|}
\hline Characteristic & $\begin{array}{l}\text { Non-operative } \\
(n=224)\end{array}$ & $\begin{array}{l}\text { SSSF } \\
(n=112)\end{array}$ & $p$-value \\
\hline Age, year, median (IQR) & $49(28)$ & $50(33)$ & 0.908 \\
\hline Male, $n(\%)$ & $179(79.9 \%)$ & $84(75.0 \%)$ & 0.303 \\
\hline ISS, median (IQR) & $19(13)$ & $19(15)$ & 0.728 \\
\hline \multicolumn{4}{|l|}{ Comorbidities, $n(\%)$} \\
\hline $\mathrm{DM}$ & $26(11.6 \%)$ & $12(10.7 \%)$ & 0.808 \\
\hline HTN & $49(21.9 \%)$ & $30(26.8 \%)$ & 0.317 \\
\hline COPD & $11(4.9 \%)$ & $10(8.9 \%)$ & 0.151 \\
\hline Smoker & $54(24.1 \%)$ & $27(24.1 \%)$ & 1 \\
\hline Chronic renal failure & $2(0.9 \%)$ & $1(0.9 \%)$ & 1 \\
\hline \multicolumn{4}{|l|}{ AIS } \\
\hline AIS head severe & $12(5.4 \%)$ & $5(4.5 \%)$ & 0.725 \\
\hline AIS thorax severe & $87(38.8 \%)$ & $42(37.5 \%)$ & 0.812 \\
\hline AIS abdomen severe & $10(4.5 \%)$ & $4(3.6 \%)$ & 0.699 \\
\hline \multicolumn{4}{|l|}{ Injuries, $n(\%)$} \\
\hline TBI & $10(4.5 \%)$ & $5(4.5 \%)$ & 1 \\
\hline Spine fracture & $20(8.9 \%)$ & $5(4.5 \%)$ & 0.142 \\
\hline Rib fracture & $28(12.5 \%)$ & $16(14.3 \%)$ & 0.647 \\
\hline Pelvis fracture & $7(3.1 \%)$ & $1(0.9 \%)$ & 0.206 \\
\hline Upper extremity fracture & $12(5.4 \%)$ & $3(2.7 \%)$ & 0.262 \\
\hline Lower extremity fracture & $16(7.1 \%)$ & $8(7.1 \%)$ & 1 \\
\hline Lung injury & $27(12.1 \%)$ & $13(11.6 \%)$ & 0.905 \\
\hline Heart/vessel injury & $1(0.4 \%)$ & $0(0 \%)$ & 0.479 \\
\hline Liver injury & $4(1.8 \%)$ & $3(2.7 \%)$ & 0.589 \\
\hline Spleen injury & $2(0.9 \%)$ & $1(0.9 \%)$ & 1 \\
\hline
\end{tabular}

SSSF Surgical stabilization of sternum fracture, NOM Non-operative management, $I Q R$ Interquartile range, ISS Injury severity score, $D M$ Diabetes mellitus, HTN Hypertension, COPD Chronic obstructive pulmonary disease, AIS Abbreviated injury scale, TBI Traumatic brain injury

matching, and Table 2 includes factors that were not controlled for with propensity matching.

\section{Primary and secondary outcomes}

Compared to patients undergoing NOM, patients undergoing SSSF had a lower mortality rate $(2.7 \%$ vs. $11.2 \%$, $p=0.008$ ) (Table 3). Considering other outcomes, The SSSF and NOM cohorts had a similar rate of ARDS $(2.7 \%$ vs. $2.2 \%, p=0.80)$, pneumonia $(1.8 \%$ vs. $0.9 \%$, $p=0.48)$, unplanned intubation $(8.9 \%$ vs. $5.8 \%, p=0.29)$ and all other complications (all $p>0.05$ ). However, SSSF patients had a longer median hospital LOS (16 vs. 7 days, $p<0.001)$, ICU LOS (9.5 vs. 5.5 days, $p=0.016)$ and ventilator days ( 8 vs. $5, p=0.035$ ).

\section{Discussion}

In this large national TQIP analysis, we found that patients with traumatic sternum fractures undergo SSSF $1.2 \%$ of the time. Despite lower associated mortality with SSSF in this study, we found no differences in any other complication between the propensity-matched SSSF and NOM cohorts. However, SSSF patients were admitted to the hospital and ICU for a longer duration and were subject to more days on the ventilator. To our knowledge, this is the first manuscript that directly compares a national group of patients managed with SSSF to patients with sternal fractures undergoing NOM.

The lower rate of mortality and no difference in complications in this study suggest that SSSF could be beneficial in select patients. This corresponds with the existing literature on SSSF which predominantly includes case studies, cohort studies, and cross-sectional studies demonstrating that both conservative and surgical approaches are safe and effective for the management of sternal fractures [1, $5,6,9,13,14]$. The increased hospital LOS and days on the ventilator associated with SSSF in our study could be due to the fact that patients were selected to undergo SSSF later in the hospitalization after conservative measures had failed. This is supported by the findings of Ciriaco et al. who found that patients undergoing SSSF were operated on anywhere from 2-6 days after admission, and patients stayed an average of three days post-operatively [9]. This suggests that the decision to pursue SSSF is often deferred several days into the hospital course [9]. An alternative explanation for the increased LOS but decreased mortality associated with SSSF could be that patients undergoing NOM have early mortality and, thus, a decreased hospital and ICU LOS, or patients undergoing SSSF have a later mortality and increased LOS. Though the two groups demonstrated a similar rate of complications, it is possible that SSSF provides patients with a superior physiologic reserve and enhanced ability to overcome complications. However, this is speculation and unable to be supported with evidence. Regardless, our findings imply that SSSF is safe and potentially effective in select patients with sternal fracture. Questions remain regarding patient selection.

The indications and timing for SSSF in the management of sternal fractures need standardization. A systematic review performed by Harston et al., found that surgeons had a variety of predominantly subjective indications regarding the decision for SSSF, such as pain, respiratory status, displacement, sternal deformity or instability, non-union, hunched posture, limited range of motion and presumed eventual pseudarthrosis with associated pain [3]. When it comes to timing for SSSF, significant variance also exists with some authors performing repair 
Table 2 Comparison of factors between patients managed with NOM vs SSSF-not propensitymatched

\begin{tabular}{|c|c|c|c|}
\hline Characteristic & $\operatorname{NOM}(n=224)$ & $\operatorname{SSSF}(n=112)$ & $p$-value \\
\hline Teaching university, $n(\%)$ & $129(57.6 \%)$ & $60(53.6 \%)$ & 0.484 \\
\hline \multicolumn{4}{|l|}{ Ethnicity, $n(\%)$} \\
\hline Black & $26(13.7 \%)$ & $11(11.8 \%)$ & 0.663 \\
\hline White & $164(73.2 \%)$ & $82(73.2 \%)$ & 1 \\
\hline Asian & $7(4.1 \%)$ & $1(1.2 \%)$ & 0.216 \\
\hline Hispanic & $27(12.6 \%)$ & $13(12.4 \%)$ & 0.964 \\
\hline \multicolumn{4}{|l|}{ Comorbidities, $n(\%)$} \\
\hline Cirrhosis & $0(0 \%)$ & $1(0.9 \%)$ & 0.157 \\
\hline Alcohol use & $48(22.0 \%)$ & $23(20.9 \%)$ & 0.157 \\
\hline CVA & $2(0.9 \%)$ & $2(1.8 \%)$ & 0.477 \\
\hline $\mathrm{CHF}$ & $4(1.8 \%)$ & $1(0.9 \%)$ & 0.524 \\
\hline \multicolumn{4}{|l|}{ AIS } \\
\hline AIS upper extremity severe & $2(0.9 \%)$ & $0(0 \%)$ & 0.316 \\
\hline AIS lower extremity severe & $2(0.9 \%)$ & $1(0.9 \%)$ & 1 \\
\hline AIS spine severe & $13(5.8 \%)$ & $6(5.4 \%)$ & 0.867 \\
\hline \multicolumn{4}{|l|}{ Injuries, $n(\%)$} \\
\hline Kidney injury & $0(0 \%)$ & $1(0.9 \%)$ & 0.157 \\
\hline GU injury & $0(0 \%)$ & $1(0.9 \%)$ & 0.157 \\
\hline Small intestine injury & $1(0.4 \%)$ & $0(0 \%)$ & 0.479 \\
\hline Colorectal injury & $2(0.9 \%)$ & $1(0.9 \%)$ & 1 \\
\hline Face/neck fracture & $5(2.2 \%)$ & $1(0.9 \%)$ & 0.382 \\
\hline \multicolumn{4}{|l|}{ Initial vital signs } \\
\hline SBP, median (IQR) & $130.5(38)$ & $129(30)$ & 0.293 \\
\hline Hypotensive on admission, $n(\%)$ & $25(11.8 \%)$ & $11(10.1 \%)$ & 0.647 \\
\hline Heart rate, median (IQR) & $95(30)$ & $97(26)$ & 0.781 \\
\hline Respiratory rate, median (IQR) & $19(6)$ & $19(7)$ & 0.921 \\
\hline \multicolumn{4}{|l|}{ Mechanism, $n(\%)$} \\
\hline Blunt vs penetrating & $35(100 \%)$ & $13(92.9 \%)$ & 0.11 \\
\hline Motorcyclist & $1(0.4 \%)$ & $1(0.9 \%)$ & 0.616 \\
\hline Pedestrian & $2(0.9 \%)$ & $0(0 \%)$ & 0.316 \\
\hline GSW & $0(0 \%)$ & $1(0.9 \%)$ & 0.157 \\
\hline Fall & $1(0.4 \%)$ & $1(0.9 \%)$ & 0.616 \\
\hline MVC & $30(13.4 \%)$ & $11(9.8 \%)$ & 0.346 \\
\hline
\end{tabular}

SSSF Surgical stabilization of sternum fracture, NOM Non-operative management, CVA Cerebrovascular accident, $C H F$ Congestive heart failure, AIS Abbreviated injury scale, $G U$ Genitourinary, SBP Systolic blood pressure, $I Q R$ Interquartile range, GSW Gunshot wound, $M V C$ Motor vehicle collision within 30 days of injury [3, 6, 7, 9, 13, 15], and others performing SSSF only on non- or malunions from 3 to 60 months post injury $[6,14,16,17]$. Though most studies do not provide detailed information regarding surgical indications, a survey performed by Mayberry et al. attempted to uncover clinical decision-making for SSSF by surveying over 400 trauma, orthopedic and thoracic surgeons [18]. Respondents were asked about indications for sternal fracture repair, and the authors found that the most agreed upon indication was non-union after 6 weeks [18]. However, only $68 \%$ of respondents agreed that this was an appropriate indication, and every other suggested indication achieved less than 50\% consensus among those surveyed [18]. With such equipoise in practice and treating philosophy, future studies are necessary-ideally a randomized controlled trial (RCT) to evaluate outcomes and indications to guide the future of SSSF.

As a retrospective database study, this analysis is susceptible to a number of limitations including missing data, reporting bias, and coding errors. We lack the ability to assess the surgeon's clinical decision-making regarding patient selection and timing of operation, and we acknowledge that there is undoubtedly selection bias regarding which patients received operative intervention. Due to the constraints of the database utilized, we are unable to pinpoint the time relationship between when a patient was placed on 
Table 3 Comparison of outcomes between patients managed with NOM and SSSF

\begin{tabular}{|c|c|c|c|}
\hline Characteristic & $\operatorname{NOM}(n=224)$ & $\operatorname{SSSF}(n=112)$ & $p$-value \\
\hline Total LOS, days, median (IQR) & $7(12)$ & $16(12)$ & $<0.001$ \\
\hline Total ICU LOS, days, median (IQR) & $5.5(9)$ & $9.5(11)$ & 0.016 \\
\hline Total vent days, median (IQR) & $5(8)$ & $8(9)$ & 0.035 \\
\hline Mortality, $n(\%)$ & $25(11.2 \%)$ & $3(2.7 \%)$ & 0.008 \\
\hline \multicolumn{4}{|l|}{ Complications, $n(\%)$} \\
\hline AKI & $5(2.2 \%)$ & $2(1.8 \%)$ & 0.787 \\
\hline ARDS & $5(2.2 \%)$ & $3(2.7 \%)$ & 0.8 \\
\hline Cardiac arrest & $12(5.4 \%)$ & $4(3.6 \%)$ & 0.469 \\
\hline Deep site infection & $1(0.4 \%)$ & $1(0.9 \%)$ & 0.616 \\
\hline DVT & $5(2.2 \%)$ & $7(6.3 \%)$ & 0.061 \\
\hline MI & $0(0 \%)$ & $1(0.9 \%)$ & 0.157 \\
\hline Organ space infection & $0(0 \%)$ & $1(0.9 \%)$ & 0.157 \\
\hline Pneumonia & $2(0.9 \%)$ & $2(1.8 \%)$ & 0.477 \\
\hline $\mathrm{PE}$ & $2(0.9 \%)$ & $4(3.6 \%)$ & 0.081 \\
\hline CVA & $1(0.4 \%)$ & $0(0 \%)$ & 0.479 \\
\hline Superficial infection & $1(0.4 \%)$ & $2(1.8 \%)$ & 0.219 \\
\hline Unplanned intubation & $13(5.8 \%)$ & $10(8.9 \%)$ & 0.285 \\
\hline UTI & $0(0 \%)$ & $1(0.9 \%)$ & 0.157 \\
\hline Unplanned return to OR & $4(1.8 \%)$ & $3(2.7 \%)$ & 0.589 \\
\hline Unplanned ICU admission & $10(4.5 \%)$ & $8(7.1 \%)$ & 0.304 \\
\hline Severe sepsis & $4(1.8 \%)$ & $2(1.8 \%)$ & 1 \\
\hline CAUTI & $5(2.2 \%)$ & $1(0.9 \%)$ & 0.382 \\
\hline VAP & $3(1.3 \%)$ & $3(2.7 \%)$ & 0.382 \\
\hline Other & $31(13.8 \%)$ & $15(13.4 \%)$ & 0.911 \\
\hline
\end{tabular}

SSSF Surgical stabilization of sternum fracture, NOM Non-operative management, LOS Length of stay, $I Q R$ Interquartile range, $I C U$ Intensive care unit, $A K I$ Acute kidney injury, ARDS Acute respiratory distress syndrome, ETOH Alcohol, DVT Deep vein thrombosis, MI Myocardial infarction, PE Pulmonary embolism, CVA Cerebrovascular accident, UTI Urinary tract infection, OR Operating room, CAUTI Catheter associated urinary tract infection, VAP Ventilator acquired pneumonia a ventilator and when the operation occurred. Particularly, we are unable to determine if some patients underwent SSSF because they were unable to wean from the ventilator or if the increased ventilator days in the SSSF group were secondary to the surgery itself. Similar questions arise when considering hospital LOS and ICU LOS and how they relate to the timing of surgery. As TQIP is limited to the index hospitalization, we cannot evaluate any post-discharge or long-term outcome differences associated with either group of patients. Finally, though this study evaluates quantitative outcomes, qualitative outcomes could not be measured, and this represents an area for future research.

\section{Conclusion}

To our knowledge, this is the first large national analysis comparing outcomes of sternum fracture patients undergoing SSSF to a similarly matched group who underwent NOM. We found that SSSF patients had a decreased mortality rate, whereas a similar rate of complications compared to NOM patients. However, SSSF patients had a longer hospital LOS and days on the ventilator. Due to a lack of guidelines pertaining to patient selection for SSSF and no RCTs in the literature, a concerted effort is needed to develop an algorithmic approach and define which patients will benefit from SSSF.

Author contributions All authors made substantial contributions to this study. Data collection and data analysis was performed by Areg Grigorian. The first draft of the manuscript was written by Ashton Christian and critical revisions were made by Jeffry Nahmias, William Duong, Michael Lekawa, Victor Joe, and Sebastian Schubl. All authors read and approved the final manuscript.

Funding The authors did not receive any funding for this research.

Data availability Data acquired from Trauma Quality Improvement Program (TQIP) between January 2015 and December 2016.

Code availability All analyses were performed with IBM SPSS Statistics for Windows (Version 24, IBM Corp., Armonk, NY). 


\section{Compliance with ethical standards}

Conflicts of interest The authors have no conflicts of interest or competing interests.

Ethical approval This study was approved by the Institutional Review Board at the University of California, Irvine.

\section{References}

1. Zhao Y, Yang Y, Gao Z, Wu W, He W, Zhao T. Treatment of traumatic sternal fractures with titanium plate internal fixation: a retrospective study. J Cardiothorac Surg. 2017;12(1):22.

2. Krinner S, Grupp S, Oppel P, Langenbach A, Hennig FF, SchulzDrost $S$. Do low profile implants provide reliable stability in fixing the sternal fractures as a "fourth vertebral column" in sternovertebral injuries? J Thorac Dis. 2017;9(4):1054-64.

3. Harston A, Roberts C. Fixation of sternal fractures: a systematic review. J Trauma. 2011;71(6):1875-9.

4. Athanassiadi K, Gerazounis M, Moustardas M, Metaxas E. Sternal fractures: retrospective analysis of 100 cases. World J Surg. 2002;26(10):1243-6.

5. Klei DS, de Jong MB, Öner FC, Leenen LP, van Wessem KJ. Current treatment and outcomes of traumatic sternal fractures: a systematic review. Int Orthop. 2018;43(6):1455-64.

6. Richardson JD, Franklin GA, Heffley S, Seligson D. Operative fixation of chest wall fractures: an underused procedure? Am Surg. 2007;73(6):591-6 (discussion 596-7).

7. Potaris K, Gakidis J, Mihos P, Voutsinas V, Deligeorgis A, Petsinis V. Management of sternal fractures: 239 cases. Asian Cardiovasc Thorac Ann. 2002;10(2):145-9.
8. Celik B, Sahin E, Nadir A, Kaptanoglu M. Sternum fractures and effects of associated injuries. Thorac Cardiov Surg. 2009;57:468-71.

9. Ciriaco P, Casiraghi M, Negri G, et al. Early surgical repair of isolated traumatic sternal fractures using a cervical plate system. J Trauma. 2009;66(2):462-4.

10. Kirkham JH. Depressed fracture of the sternum. Br Med J. 1941;2(4217):614.

11. Brin YS, Palmanovich E, Dolev E, Nyska M, Kish BJ. Displaced mid-shaft clavicular fractures: is conservative treatment still preferred? Isr Med Assoc J. 2014;16(12):748-52.

12. Canadian Orthopaedic Trauma Society. Nonoperative treatment compared with plate fixation of displaced midshaft clavicular fractures. A multicenter, randomized clinical trial. J Bone Joint Surg Am. 2007;89(1):1-10.

13. Al-Qudah A. Operative treatment of sternal fractures. Asian Cardiovasc Thorac Ann. 2006;14:399-401.

14. Wu LC, Renucci JD, Song DH. Sternal nonunion: a review of current treatments and a new method of rigid fixation. Ann Plast Surg. 2005;54:55-8.

15. Kitchens J, Richardson JD. Open fixation of sternal fracture. Surg Gynecol Obstet. 1993;177:423-4.

16. Molina JE. Evaluation and operative technique to repair isolated sternal fracture. J Thorac Cardiovasc Surg. 2005;130:445-8.

17. Coons DA, Pitcher JD, Braxton M, Bickley BT. Sternal nonunion, case report. Orthopedics. 2002;25:89-91.

18. Mayberry JC, Ham LB, Schipper PH, Ellis TJ, Mullins RJ. Surveyed opinion of American trauma, orthopedic, and thoracic surgeons on rib and sternal fracture repair. J Trauma. 2009;66:875-9. 